\title{
Fault Detection for Linear Discrete Time-Varying Systems with Measurement Packet Dropping
}

\author{
Yueyang Li, ${ }^{1}$ Shuai Liu, ${ }^{2,3}$ and Zhonghua Wang ${ }^{1}$ \\ ${ }^{1}$ School of Electrical Engineering, University of Jinan, Jinan 250022, China \\ ${ }^{2}$ School of Control Science and Engineering, Shandong University, Jinan 250061, China \\ ${ }^{3}$ School of Electrical and Electronic Engineering, Nanyang Technological University, Singapore 639798
}

Correspondence should be addressed to Yueyang Li; cse_liyy@ujn.edu.cn

Received 24 January 2013; Accepted 8 April 2013

Academic Editor: Wuquan Li

Copyright (C) 2013 Yueyang Li et al. This is an open access article distributed under the Creative Commons Attribution License, which permits unrestricted use, distribution, and reproduction in any medium, provided the original work is properly cited.

\begin{abstract}
The fault detection (FD) problem for linear discrete time-varying (LDTV) systems with measurement packet dropouts is considered. The objective is to design a new observer-based fault detection filter (FDF) as a residual generator through employing packet dropout information on the measurement sequence. Based on some new defined input-to-output operators, the FD problem is formulated in a framework of maximizing stochastic $H_{-} / H_{\infty}$ or $H_{\infty} / H_{\infty}$ performance index. By introducing an adjoint-operatorbased optimization method, the analytical optimal solution can be derived in terms of solving a modified Riccati equation. A numerical example is provided to demonstrate the effectiveness of the proposed approach.
\end{abstract}

\section{Introduction}

The problem of model-based fault detection and isolation has attracted much attention during the last two decades and bred numerous results. One of the most useful strategies is the observer-based fault detection (FD) approach which involves constructing an observer as a fault detection filter (FDF) for generating a residual signal that is sensitive to fault while insensitive to unknown input [1-7]. Among the developments of FD techniques, the $H_{\infty}$ filtering scheme which formulates the FD problem into the framework of $H_{\infty}$ filtering is widely used for systems with $\mathscr{L}_{2}$-norm bounded unknown inputs and faults [8-11]. Another efficient way is the so-called $H_{\infty}$ optimization scheme which employs $H_{\infty}$ norm as a robustness measure while the $H_{\infty}$ or $H_{-}$ norm is introduced as a fault sensitivity measure, and, then, the FD problem can be formulated as an $H_{\infty} / H_{\infty}$ or $H_{-} / H_{\infty}$ optimization problem. In [12-14], the global optimal solution to $H_{\infty} / H_{\infty}$ and $H_{-} / H_{\infty}$ optimization FD problem is given for linear time-invariant (LTI) systems by coprime factorization and linear matrix inequality (LMI) techniques, respectively. Recent years, some new results are dedicated to this problem for linear time-varying (LTV) systems, especially for linear discrete time-varying (LDTV) systems, under the background that real systems are intrinsically time varying and most industrial processes are operated by digital devices. In $[15,16]$, the unified approach in [12] is extended to $H_{\infty} / H_{\infty}$ optimization FD problem for linear discrete-time periodic systems. Finite horizon $H_{-} / H_{\infty}$ and $H_{\infty} / H_{\infty}$ FD formulation for LDTV systems is proposed in [17-19] and the optimal solution is derived by solving a standard Kalman-like Riccati equation.

On another frontline, research on systems with intermittent measurements has gained growing interests due to that the communication networks are widely utilized in control systems [20-25]. In the literature of control and filtering, stochastic parameters such as the Bernoulli distributed variables and Markov chain are introduced to model the phenomenon of missing measurements, which can be divided into two fundamentally different categories. One is the UDP-like protocol-based model where there is no acknowledgement mechanism of successful delivery of data packets. The other model is based on the TCP-like protocol where successful transmissions of packets are acknowledged at the receiver [20]. With the increasing demands for system safety and reliability, FD for systems with measurement 
packet dropping has become one of the most critical issues and many related results have emerged with the help of LMI technique. In [26], a robust $H_{\infty}$ FDF is designed for LTI systems with missing measurements. In [27-29], the same idea is extended to Markovian jump singular systems, TS fuzzy systems, and discrete-time switched systems under intermittent measurements condition, respectively. The $H_{\infty}$ filtering FD scheme is also applied to networked control systems with both induced delays and incomplete measurements through augmenting retarded vectors into delay-free variables and numerical solutions are derived, we refer to [30-34] and references therein. However, it should be noted that most of the existing results are based on $H_{\infty}$ filtering scheme under the assumption that the measurements are transmitted using UDP-like protocol-based model and only infinite-horizon cases are considered. There remain some problems to be figured out in the open area; for example, the existing LMIbased approach does not efficiently work for time-varying systems owing to large computational burden brought by optimization iterative algorithm at each time instant, and, consequently, how to detect a fault for LDTV systems with missing measurements in the TCP-like protocols scenario.

Motivated by the above discussions, the FD problem for LDTV systems with measurement packet dropout in TCPlike protocol frame will be investigated in this paper. The main contributions of the paper are twofold.

(1) A more generalized FD problem description in the framework of optimizing stochastic $H_{-} / H_{\infty}$ or $H_{\infty} / H_{\infty}$ performance index is formulated by defining input-to-output operator that maps from fault or unknown input to residual signal. Then, a new observer-based FDF is proposed for generating residuals based on the acknowledgement mechanism of data packet transmission.

(2) An analytical solution to the aforementioned problem is explicitly expressed by solving a modified Riccati equation through a proposed adjoint-operator-based optimization method.

The remainder of this paper is organized as follows. In Section 2, the FD problem is formulated in the sense of maximizing a stochastic sensitivity/robustness ratio. In Section 3, a unified solution to optimizing stochastic $H_{-} / H_{\infty}$ or $H_{\infty} / H_{\infty}$ performance index problem is derived by using the proposed adjoint-operator-based optimization scheme. Finally, an illustrative example is given in Section 4 to demonstrate the effectiveness of our proposed approach.

Notations. Throughout this paper, for a matrix $X, X^{\mathrm{T}}$ and $X^{-1}$ stand for the transpose and inverse of $X$, respectively. $R^{n}$ means the set of $n$-dimensional real vectors. $I$ and 0 denote identity matrix and zero matrix with appropriate dimensions, respectively. $X>0(X<0)$ means that $X$ is positive (negative) definite. $\mathrm{E}\{\vartheta(k)\}$ represents the mathematical expectation of $\vartheta(k)$. $\|\xi\|$ denotes the Euclidean norm of $\xi$. $\|\theta(k)\|_{2}$ stands for the deterministic $l_{2}$-norm of $\theta(k)$ with $\|\theta(k)\|_{2}^{2}=$ $\sum_{k=0}^{N} \theta^{\mathrm{T}}(k) \theta(k)$, while $\|\zeta(k)\|_{2, \mathrm{E}}$ for the stochastic case with $\|\zeta(k)\|_{2, \mathrm{E}}^{2}=\mathrm{E}\left\{\sum_{k=0}^{N} \zeta^{\mathrm{T}}(k) \zeta(k)\right\}$, where $N$ is a positive integer. $\left\langle\left(\mu_{0}, \mu(k)\right),\left(\varsigma_{0}, \varsigma(k)\right)\right\rangle=\mathrm{E}\left\{\mu_{0}^{\mathrm{T}} \Pi \varsigma_{0}\right\}+\mathrm{E}\left\{\sum_{k=0}^{N} \mu^{\mathrm{T}}(k) \varsigma(k)\right\}$ gives the definition of the inner product on a Hilbert space for $l_{2}$-norm bounded vector $\mu(k)$ and $\varsigma(k)$ with appropriate dimensions, where $\Pi$ is a positive-definite initial weighting matrix. Prob $\{\cdot\}$ means the occurrence probability of the event “.”.

\section{Problem Formulation}

Consider the following LDTV system with measurement packet dropping:

$$
\begin{gathered}
x(k+1)=A(k) x(k)+B(k) u(k) \\
+B_{d}(k) d(k)+B_{f}(k) f(k), \\
y(k)=\gamma(k) C(k) x(k)+D_{d}(k) d(k), \\
x(0)=x_{0},
\end{gathered}
$$

where $x(k) \in R^{n}, y(k) \in R^{n_{y}}, u(k) \in R^{n_{u}}, d(k) \in R^{n_{d}}$, and $f(k) \in R^{n_{f}}$ denote the state, measurement output, control input, unknown input, and fault, respectively. $A(k), B(k), B_{d}(k), B_{f}(k), C(k)$, and $D_{d}(k)$ are known timevarying matrices with appropriate dimensions. $f(k)$ and $d(k)$ are $l_{2}$-norm bounded signals. $\gamma(k)$ depicts the measurement packet dropouts and is assumed to be a scalar Bernoulli distributed binary stochastic variable; that is,

$$
\begin{aligned}
& \operatorname{Prob}\{\gamma(k)=1\}=\mathrm{E}\{\gamma(k)\}=\rho, \\
& \operatorname{Prob}\{\gamma(k)=0\}=1-\mathrm{E}\{\gamma(k)\}=1-\rho,
\end{aligned}
$$

where $\rho$ is a known constant which can be obtained as prior knowledge through statistical test method [32].

Before describing the underlying problems, the following definition and assumptions are introduced.

Definition 1. System (1) is said exponentially stable in mean square sense (ESMS) with $u(k)=0, d(k)=0$, and $f(k)=0$, if there exist $\beta \in(0,+\infty)$ and $q \in(0,1)$ such that

$$
\mathrm{E}\left\{\|x(k)\|^{2}\right\} \leq \beta q^{k}\|x(0)\|^{2},
$$

for any initial condition $x_{0}$.

Assumption 2. $\left(A^{\mathrm{T}}(k), \rho C^{\mathrm{T}}(k), 0, C^{\mathrm{T}}(k)\right)$ is stabilizable.

Assumption 3. $\left(A^{\mathrm{T}}(k), 0, B_{d}^{\mathrm{T}}(k)\right)$ is exactly observable.

Assumption 4. $\gamma(k)$ is available at each time instant $k$.

The primary object of FD is to construct an FDF for generating a residual signal which is robust to unknown input $d(k)$ while sensitive to fault $f(k)$. Note that the indicator 
$\gamma(k)$ is known due to Assumption 4, and hence $\gamma(k)$ can be involved in the following observer-based FDF:

$$
\begin{gathered}
\widehat{x}(k+1)=A(k) \hat{x}(k)+B(k) u(k) \\
+L(k)(y(k)-\gamma(k) C(k) \hat{x}(k)), \\
r(k)=V(k)(y(k)-\gamma(k) C(k) \hat{x}(k)), \\
\widehat{x}(0)=\widehat{x}_{0},
\end{gathered}
$$

where $\widehat{x}(k) \in R^{n}$ is an estimation of $x(k), r(k) \in R^{r}$ is the generated residual, $\widehat{x}_{0}$ is a guess of initial state, $L(k)$ is the observer gain matrices, and $V(k)$ is the (regular) post-filters to be determined.

$$
\begin{aligned}
\text { Let } e(k)= & x(k)-\widehat{x}(k) ; \text { it follows from }(1) \text { and }(4) \text { that } \\
e(k+1)= & (A(k)-\gamma(k) L(k) C(k)) e(k) \\
& +\left(B_{d}(k)-L(k) D_{d}(k)\right) d(k)+B_{f}(k) f(k), \\
r(k)= & V(k)\left(\gamma(k) C(k) e(k)+D_{d}(k) d(k)\right) .
\end{aligned}
$$

Denote

$$
\begin{aligned}
& r_{f}(k)=\left.r(k)\right|_{d_{k}=0}, \\
& r_{d}(k)=\left.r(k)\right|_{f_{k}=0},
\end{aligned}
$$

where $d_{k}=\left[\begin{array}{llll}e^{\mathrm{T}}(0) & d^{\mathrm{T}}(0) & \cdots & d^{\mathrm{T}}(k)\end{array}\right]^{\mathrm{T}}$ and $f_{k}=$ $\left[\begin{array}{lll}f^{\mathrm{T}}(0) & \cdots & f^{\mathrm{T}}(k)\end{array}\right]^{\mathrm{T}}$.

Similar to the perturbed operator defined by $l_{2}$-norm of input and output signals in [35], a linear operator that maps $f \mapsto r$ and a linear operator that maps $d \mapsto r$ can be defined as follows by the linearity of (1):

$$
\begin{gathered}
r_{f}(k)=\mathscr{G}_{r f} f(k), \\
r_{d}(k)=\mathscr{G}_{r d} d(k)
\end{gathered}
$$

with

$$
\begin{gathered}
\left\|\mathscr{G}_{r f}\right\|_{\infty}=\sup _{f \in l_{2},\|f\|_{2} \neq 0} \frac{\left\|r_{f}(k)\right\|_{2, \mathrm{E}}^{2}}{\|f(k)\|_{2}^{2}}, \\
\left\|\mathscr{G}_{r d}\right\|_{\infty}=\sup _{d \in l_{2},\|d\|_{2} \neq 0} \frac{\left\|r_{d}(k)\right\|_{2, \mathrm{E}}^{2}}{\|d(k)\|_{2}^{2}+e^{\mathrm{T}}(0) S e(0)}, \\
\left\|\mathscr{G}_{r f}\right\|_{-}=\inf _{f \in l_{2},\|f\|_{2} \neq 0} \frac{\left\|r_{f}(k)\right\|_{2, \mathrm{E}}^{2}}{\|f(k)\|_{2}^{2}},
\end{gathered}
$$

where $S$ is a positive-definite initial weighting matrix.

Based on the definitions above, we are now in the position to introduce an auxiliary FDF design problem for residual generation: find a suitable observer gain matrix $L(k)$ and a regular post-filter $V(k)$ such that system (5) is ESMS and satisfies the following performance:

$$
\max _{L(k), V(k)} \frac{\left\|\mathscr{G}_{r f}\right\|_{\infty}}{\left\|\mathscr{G}_{r d}\right\|_{\infty}} \text { or } \max _{L(k), V(k)} \frac{\left\|\mathscr{G}_{r f}\right\|_{-}}{\left\|\mathscr{G}_{r d}\right\|_{\infty}} .
$$

After designing the FDF, the remaining task is to evaluate the generated residual. In this paper, we adopt the residual evaluation function in the following forms:

$$
J(k)=\sqrt{\frac{1}{k} \sum_{k=0}^{k=k_{T}} r^{\mathrm{T}}(k) r(k),}
$$

where $k_{T}$ denotes the length of the evaluation time window [2]. The corresponding threshold is chosen as

$$
J_{\text {th }}=\sup _{f(k)=0} \mathrm{E}\{J(k)\} \text {, }
$$

and hence the occurrence of faults can then be recognized based on the following rule:

$$
\begin{aligned}
& J(k)>J_{\mathrm{th}}, \quad \Longrightarrow \text { a fault is detected, } \\
& J(k) \leq J_{\mathrm{th}}, \quad \Longrightarrow \text { no faults. }
\end{aligned}
$$

Remark 5. Assumptions 2 and 3 are given without loss of generality to guarantee the stability of the proposed FDF. For more details about the definitions on stabilizable and exactly observable, we refer to $[24,36]$ and references therein. With the aid of time-stamp technique, Assumption 4 is also reasonable due to the acknowledgements of successful transmissions of data packets for TCP-like protocols. Furthermore, under the condition that Assumption 4 holds, our proposed FDF is different from the ones given in [26-29] since the new FDF exploits additional information on the observation sequence.

Remark 6. It should be emphasized that since stochastic characteristic is introduced by $\gamma(k)$, the existing FD problem formulation and results in [17-19] for deterministic LTV systems would not be reasonably established. Our proposed performance index $\left\|\mathscr{G}_{r f}\right\|_{\infty} /\left\|\mathscr{G}_{r d}\right\|_{\infty}$ or $\left\|\mathscr{G}_{r d}\right\|_{-} /\left\|\mathscr{G}_{r d}\right\|_{\infty}$ is a generalized stochastic version comparing to that of the existing contributions. In what follows, a novel adjointoperator-based optimization approach will be proposed with the well-defined linear operator $\mathscr{G}_{r d}$ and $\mathscr{G}_{r f}$, and we will show that detailed interpretation of $\mathscr{G}_{r d}$ and $\mathscr{G}_{r f}$ is not necessary owing to our proposed method.

\section{Main Results}

Before deriving the main results of this paper, the following definitions and lemmas should be given.

Definition 7 (see [37]). Let $\mathscr{G}_{s}$ denote an operator or a system mapping from $l_{2}$-norm bounded space $S_{1}$ to $l_{2}$-norm bounded space $S_{2}$. An operator $\mathscr{G}_{s}^{\sim}$ is called to be the adjoint operator of $\mathscr{G}_{s}$ from space $S_{2}$ to $S_{1}$ if $\left\langle\mathscr{G}_{s}\left(\mu_{0}, \mu\right), \varsigma\right\rangle=$ $\left\langle\left(\mu_{0}, \mu\right), \mathscr{G}_{s}^{\sim} \varsigma\right\rangle$, for all $\mu \in S_{1}$ and $\varsigma \in S_{2}$, where $\mu_{0}$ stands for the initial vector.

Definition 8 (see [38]). Let $\mathscr{G}_{s}$ denote an operator or a system mapping from $l_{2}$-norm bounded input space $S_{1}$ to $l_{2}$-norm bounded output space $S_{2}$; then, $\mathscr{G}_{s}$ is said to be coisometric if $\left\langle\mathscr{G}_{s}^{\sim} \varphi(k), \mathscr{G}_{s}^{\sim} \varphi(k)\right\rangle=\langle\varphi(k), \varphi(k)\rangle$ for all $\varphi(k) \in S_{1}$. 
Lemma 9. For two stochastic operators $A: y \mapsto z$ and $B$ : $w \mapsto y$, where $y, z$, and $w$ are $l_{2}$-norm bounded signals, one has

$$
\begin{gathered}
\|A B\|_{\infty} \leq\|A\|_{\infty}\|B\|_{\infty}, \\
\|A B\|_{-} \leq\|A\|_{\infty}\|B\|_{-} .
\end{gathered}
$$

Proof. Let $z=A y$ and $y=B w$; we can conclude that

$$
\begin{aligned}
\|A B\|_{\infty} & =\sup _{w \in l_{2},\|w\|_{2} \neq 0} \frac{\|z(k)\|_{2, \mathrm{E}}^{2}}{\|w(k)\|_{2}^{2}}=\sup _{w \in l_{2},\|w\|_{2} \neq 0} \frac{\|A B w(k)\|_{2, \mathrm{E}}^{2}}{\|w(k)\|_{2}^{2}} \\
& =\sup _{w \in l_{2},\|w\|_{2} \neq 0}\left\{\frac{\|A B w(k)\|_{2, \mathrm{E}}^{2}}{\|B w(k)\|_{2}^{2}} \times \frac{\|B w(k)\|_{2, \mathrm{E}}^{2}}{\|w(k)\|_{2}^{2}}\right\} \\
& \leq\|A\|_{\infty}\|B\|_{\infty}, \\
\|A B\|_{-} & =\inf _{w \in l_{2},\|w\|_{2} \neq 0} \frac{\|z(k)\|_{2, \mathrm{E}}^{2}}{\|w(k)\|_{2}^{2}}=\inf _{w \in l_{2},\|w\|_{2} \neq 0} \frac{\|A B w(k)\|_{2, \mathrm{E}}^{2}}{\|w(k)\|_{2}^{2}} \\
& =\inf _{w \in l_{2},\|w\|_{2} \neq 0}\left\{\frac{\|z(k)\|_{2, \mathrm{E}}^{2}}{\|y(k)\|_{2}^{2}} \times \frac{\|B w(k)\|_{2, \mathrm{E}}^{2}}{\|w(k)\|_{2}^{2}}\right\} \\
& \leq \sup _{w \in l_{2},\|w\|_{2} \neq 0} \frac{\|z(k)\|_{2, \mathrm{E}}^{2}}{\|y(k)\|_{2}^{2}} \times \inf _{w \in l_{2},\|w\|_{2} \neq 0} \frac{\|B w(k)\|_{2, \mathrm{E}}^{2}}{\|w(k)\|_{2}^{2}} \\
& \leq\|A\|_{\infty}\|B\|_{-}
\end{aligned}
$$

which completes the proof.

Lemma 10. Consider the following residual generators:

$$
\begin{aligned}
\hat{x}^{(m)}(k+1)= & A(k) \hat{x}^{(m)}(k)+B(k) u(k) \\
& +L^{(m)}(k)\left(y(k)-\gamma(k) C(k) \hat{x}^{(m)}(k)\right), \\
r^{(m)}(k)= & V^{(m)}(k)\left(y(k)-\gamma(k) C(k) \hat{x}^{(m)}(k)\right), \\
& m=1,2,
\end{aligned}
$$

where $L^{(m)}(k)$ is the observer gain matrix such that system (15) is ESMS and $V^{(m)}(k)$ is the post-filter. Then

$$
r^{(2)}(k)=Q r^{(1)}(k)
$$

where $Q$ is an operator that maps $r^{(1)}(k) \mapsto r^{(2)}(k)$.

Proof. By applying Lemma 1 in [18] to (15), we have that for the following residual generators

$$
\begin{aligned}
\widehat{x}^{(m)}(k+1)= & A(k) \hat{x}^{(m)}(k)+B(k) u(k) \\
& +L^{(m)}(k)\left(y(k)-\gamma(k) C(k) \widehat{x}^{(m)}(k)\right), \\
\varepsilon^{(m)}(k)= & y(k)-\gamma(k) C(k) \hat{x}^{(m)}(k), \quad m=1,2,
\end{aligned}
$$

where $L^{(m)}(k)$ is the observer gain matrix that ensures the stability of (15); an operator $\widehat{Q}_{\varepsilon}$ that guarantees $\varepsilon^{(2)}(k)=$ $Q_{\varepsilon} \varepsilon^{(1)}(k)$ exists, which can be realized as

$$
\begin{aligned}
\xi(k+1)= & \left(A(k)-L^{(2)}(k) \gamma(k) C(k)\right) \xi(k) \\
& +\left(L^{(1)}(k)-L^{(2)}(k)\right) \nu(k), \\
\varepsilon^{Q_{\varepsilon}}(k)= & \gamma(k) C(k) \xi(k)+\nu(k) .
\end{aligned}
$$

The regularity of $V(k)$ indicates there exists $V^{+}(k)$ such that

$$
V^{+}(k) V(k)=I
$$

where $V^{+}(k)$ denotes the left inverse of $V(k)$, and then we have

$$
\begin{aligned}
r^{(2)}(k) & =V^{(2)}(k) \varepsilon^{(2)}(k)=V^{(2)}(k) \mathcal{Q}_{\varepsilon} \varepsilon^{(1)}(k) \\
& =V^{(2)}(k) \mathbb{Q}_{\varepsilon}\left(V^{(1)}(k)\right)^{+} r^{(1)}(k) .
\end{aligned}
$$

Thus, from (18), Q can be represented as

$$
\begin{aligned}
\eta(k+1)= & \left(A(k)-L^{(2)}(k) \gamma(k) C(k)\right) \xi(k) \\
& +\left(L^{(1)}(k)-L^{(2)}(k)\right) \nu(k), \\
r^{(2)}(k)= & V^{(2)}(k)(\gamma(k) C(k) \xi(k)+\nu(k)),
\end{aligned}
$$

where $v(k)=V^{(2)}(k) \mathcal{Q}_{\varepsilon}\left(V^{(1)}(k)\right)^{+} r^{(1)}(k)$. This completes the proof.

Lemma 11. For system (5), consider the operator $\mathscr{G}_{r d}$ that maps $d(k) \mapsto r_{d}(k)$ which is realized by the following discretetime system:

$$
\begin{gathered}
e(k+1)=A_{e}(k) e(k)+B_{e}(k) d(k) \\
r_{d}(k)=C_{e}(k) e(k)+D_{e}(k) d(k) \\
e(0)=e_{0}
\end{gathered}
$$

where $A_{e}(k)=A(k)-\gamma(k) L(k) C(k), B_{e}(k)=B_{d}(k)-$ $L(k) D_{d}(k), C_{e}(k)=\gamma(k) V(k) C(k)$, and $D_{e}(k)=V(k) D_{d}(k)$. Let $\mathscr{G}_{r d}^{\sim}$ be the adjoint operator of $\mathscr{G}_{r d}$; then $\mathscr{G}_{r d}$ is coisometric 
if there exists a positive definite matrix $P(\cdot)$ satisfying the following equation:

$$
\begin{aligned}
& P(k+1)= B_{e}(k) B_{e}^{\mathrm{T}}(k)+A(k) P(k) A^{\mathrm{T}}(k) \\
&+\rho L(k) C(k) P(k) C^{\mathrm{T}}(k) L^{\mathrm{T}}(k) \\
&-\rho L(k) C(k) P(k) A^{\mathrm{T}}(k) L^{\mathrm{T}}(k) \\
&-\rho A(k) C(k) P(k) C^{\mathrm{T}}(k) L^{\mathrm{T}}(k), \\
& 0= \rho A(k) P(k) C^{\mathrm{T}}(k) V^{\mathrm{T}}(k) \\
&-\rho L(k) C(k) P(k) C^{\mathrm{T}}(k) V^{\mathrm{T}}(k) \\
&+B_{e}(k) D_{e}^{\mathrm{T}}(k), \\
& I=D_{e}(k) D_{e}^{\mathrm{T}}(k)+\rho V(k) C(k) P(k) C^{\mathrm{T}}(k) V^{\mathrm{T}}(k), \\
& \quad P(0)=S^{-1} .
\end{aligned}
$$

Proof. Denote $\mathscr{G}_{r d}^{\sim} r_{d}(k)=\left[\begin{array}{ll}\eta^{\mathrm{T}}(0) & d_{a}^{\mathrm{T}}(k)\end{array}\right]^{\mathrm{T}}$. From [39], the state space representation of $\mathscr{G}_{r d}^{\sim}$ can be obtained as

$$
\begin{gathered}
\eta_{a}(k-1)=A_{e}^{\mathrm{T}}(k) \eta_{a}(k)+C_{e}^{\mathrm{T}}(k) r_{d}(k), \\
d_{a}(k)=B_{e}^{\mathrm{T}}(k) \eta_{a}(k)+D_{e}^{\mathrm{T}}(k) r_{d}(k), \\
\eta_{a}(N)=0 .
\end{gathered}
$$

For (24), define

$$
\mathbb{V}\left(\eta_{a}(k), k\right)=\eta_{a}^{\mathrm{T}}(k) P(k+1) \eta_{a}(k), \quad P(\cdot)>0 .
$$

Moreover, based on (22) and (24), we have

$$
\begin{aligned}
& \mathrm{E}\left\{\sum_{k=0}^{N} d_{a}^{\mathrm{T}}(k) d_{a}(k)\right\}+\eta_{a}^{\mathrm{T}}(-1) S^{-1} \eta_{a}(-1) \\
& =\mathrm{E}\left\{\sum_{k=0}^{N} d_{a}^{\mathrm{T}}(k) d_{a}(k)+\mathbb{V}\left(\eta_{a}(k-1), k-1\right)\right. \\
& \left.\quad-\mathbb{V}\left(\eta_{a}(k), k\right)\right\}+\eta_{a}(-1)^{\mathrm{T}} S^{-1} \eta_{a}(-1) \\
& +\eta_{a}(N)^{\mathrm{T}} P(N+1) \eta_{a}(N)-\eta_{a}(-1)^{\mathrm{T}} P(0) \eta_{a}(-1) \\
& =\mathrm{E}\left\{\sum _ { k = 0 } ^ { N } \eta _ { a } ^ { \mathrm { T } } ( k ) \left[B_{e}(k) B_{e}^{\mathrm{T}}(k)+A_{e}(k) P(k) A_{e}^{\mathrm{T}}(k)\right.\right. \\
& \quad-P(k+1)] \eta_{a}(k)+2 \eta_{a}^{\mathrm{T}}(k) \\
& \quad \times\left[B_{e}(k) D_{e}^{\mathrm{T}}(k)+A_{e}(k) P(k) C_{e}^{\mathrm{T}}(k)\right] r_{d}(k) \\
& \left.+r_{d}^{\mathrm{T}}\left[D_{e}(k) D_{e}^{\mathrm{T}}(k)+C_{e}(k) P(k) C_{e}^{\mathrm{T}}(k)\right] r_{d}(k)\right\} .
\end{aligned}
$$

Remembering that $\mathrm{E}\{\gamma(k)\}=\rho$ and $\mathrm{E}\left\{\gamma^{2}(k)\right\}=\rho$, then, from (26) and Definition 8, if

$$
\left\langle\left(\eta(0), d_{a}(k)\right),\left(\eta(0), d_{a}(k)\right)\right\rangle=\left\langle r_{d}(k), r_{d}(k)\right\rangle=\left\|r_{d}\right\|_{2, \mathrm{E}}^{2} ;
$$

that is, (23) holds, $\mathscr{G}_{r d}$ is coisometric, which completes the proof.

In view of the proposed lemmas above, we now present solutions to the optimization problem (9).

Theorem 12. If Assumptions 2 and 3 hold, then the following matrix pair

$$
\begin{gathered}
L_{o}(k)=\left(\rho A(k) P(k) C^{\mathrm{T}}(k)+B_{d}(k) D_{d}^{\mathrm{T}}(k)\right) R(k)^{-1}, \\
V_{o}(k)=R(k)^{-1 / 2}
\end{gathered}
$$

with

$$
R(k)=\rho C(k) P(k) C^{\mathrm{T}}(k)+D_{d}(k) D_{d}^{\mathrm{T}}(k)>0
$$

give a solution to the auxiliary FDF design problem and $P(k)$ is the solution to the following equation with $P(0)=S^{-1}$ :

$$
\begin{aligned}
P(k+1)= & A(k) P(k) A^{\mathrm{T}}(k) \\
& -L(k) R(k) L^{\mathrm{T}}(k)+B_{d}(k) B_{d}^{\mathrm{T}}(k) .
\end{aligned}
$$

Proof. We first give the proof of the optimal solution to (9). Let $r_{o}(k)$ be the optimal generated residual in the sense of (9). Since system (1) is linear, by applying Lemma 10 , we know that there exists an operator $Q_{r}$ such that

$$
\begin{aligned}
r(k) & =r_{f}(k)+r_{d}(k)=\mathscr{G}_{r f} f(k)+\mathscr{G}_{r d} d(k) \\
& =Q_{r} r_{o}(k)=Q_{r}\left(r_{o, f}(k)+r_{o, d}(k)\right),
\end{aligned}
$$

where $r_{o, f}(k)=\left.r_{o}(k)\right|_{d_{k}=0}$ and $r_{o, d}(k)=\left.r_{o}(k)\right|_{f_{k}=0}$, which shows that

$$
r_{d}(k)=\mathbb{Q}_{r} r_{o, d}(k) .
$$

On the other hand, consider the operator $\mathscr{G}_{r d}$ that maps $d \mapsto$ $r$ in (5); we have

$$
r_{o, d}(k)=\mathscr{G}_{r d, o} d(k),
$$

where $\mathscr{G}_{r d, o}=\left.\mathscr{G}_{r d}\right|_{L(k)=L_{o}(k), V(k)=V_{o}(k)}$, which concludes that

$$
\mathscr{G}_{r d}=\mathbb{Q}_{r} \mathscr{G}_{r d, o}
$$

Similarly, we have

$$
\mathscr{G}_{r f}=Q_{r} \mathscr{G}_{r f, o},
$$

where $\mathscr{G}_{r f, o}=\left.\mathscr{G}_{r f}\right|_{L(k)=L_{o}(k), V(k)=V_{o}(k)}$. Hence,

$$
\frac{\left\|\mathscr{G}_{r f}\right\|_{\infty}}{\left\|\mathscr{G}_{r d}\right\|_{\infty}}=\frac{\left\|\mathcal{Q}_{r} \mathscr{G}_{r f, o}\right\|_{\infty}}{\left\|\mathcal{Q}_{r} \mathscr{G}_{r d, o}\right\|_{\infty}} .
$$


Based on (24) and according to $[40,41]$ and Definition 8; that is, if $\mathscr{G}_{r d, o}$ is coisometric, we have that

$$
\begin{aligned}
\left\|\mathscr{G}_{r d}\right\|_{\infty} & =\left\|\mathscr{G}_{r d}^{\sim}\right\|_{\infty}=\sup _{r_{d} \in l_{2},\left\|r_{d}\right\|_{2, \mathrm{E}} \neq 0} \frac{\left\langle d_{a}(k), d_{a}(k)\right\rangle}{\left\|r_{d}(k)\right\|_{2, \mathrm{E}}^{2}} \\
& =\sup _{r_{d} \in l_{2},\left\|r_{d}\right\|_{2, \mathrm{E}} \neq 0} \frac{\left\langle\left(\mathcal{Q}_{r} \mathscr{G}_{r d, o}\right)^{\sim} r_{d}(k),\left(\mathcal{Q}_{r} \mathscr{G}_{r d, o}\right)^{\sim} r_{d}(k)\right\rangle}{\left\|r_{d}(k)\right\|_{2, \mathrm{E}}^{2}} \\
& =\sup _{r_{d} \in l_{2},\left\|r_{d}\right\|_{2, \mathrm{E}} \neq 0} \frac{\left\langle r_{d}(k), Q_{r} \mathscr{G}_{r d, o} \mathscr{G}_{r d, o}^{\sim} \mathcal{Q}_{r}^{\sim} r_{d}(k)\right\rangle}{\left\|r_{d}(k)\right\|_{2, \mathrm{E}}^{2}} \\
& =\left\|\mathbb{Q}_{r}\right\|_{\infty}
\end{aligned}
$$

and the following inequality is immediately established based on Lemma 9:

$$
\frac{\left\|\mathscr{G}_{r f}\right\|_{\infty}}{\left\|\mathscr{G}_{r d}\right\|_{\infty}}=\frac{\left\|\mathcal{Q}_{r} \mathscr{G}_{r f, o}\right\|_{\infty}}{\left\|\mathscr{Q}_{r} \mathscr{G}_{r d, o}\right\|_{\infty}} \leq \frac{\left\|\mathscr{Q}_{r}\right\|_{\infty}\left\|\mathscr{G}_{r f, o}\right\|_{\infty}}{\left\|\mathscr{Q}_{r}\right\|_{\infty}}=\left\|\mathscr{G}_{r f, o}\right\|_{\infty}
$$

which gives the optimal value of maximizing the performance index $\left\|\mathscr{G}_{r f}\right\|_{\infty} /\left\|\mathscr{G}_{r d}\right\|_{\infty}$.

Following the same idea, we can prove that

$$
\frac{\left\|\mathscr{G}_{r f}\right\|_{-}}{\left\|\mathscr{G}_{r d}\right\|_{\infty}}=\frac{\left\|\mathcal{Q}_{r} \mathscr{G}_{r f, o}\right\|_{-}}{\left\|\mathcal{Q}_{r} \mathscr{G}_{r d, o}\right\|_{\infty}} \leq \frac{\left\|\mathcal{Q}_{r}\right\|_{\infty}\left\|\mathscr{G}_{r f, o}\right\|_{-}}{\left\|Q_{r}\right\|_{\infty}}=\left\|\mathscr{G}_{r f, o}\right\|_{-} .
$$

Furthermore, from Lemma 11, by solving the following equations in (23):

$$
\begin{aligned}
\rho A(k) & P(k) C^{\mathrm{T}}(k) V^{\mathrm{T}}(k)-\rho L(k) C(k) P(k) C^{\mathrm{T}}(k) V^{\mathrm{T}}(k) \\
+ & B_{e}(k) D_{e}^{\mathrm{T}}(k)=0, \\
& D_{e}(k) D_{e}^{\mathrm{T}}(k)+\rho V(k) C(k) P(k) C^{\mathrm{T}} V^{\mathrm{T}}=I
\end{aligned}
$$

we can obtain (28) and consequently

$$
\begin{gathered}
P(k+1)=B_{e}(k) B_{e}^{\mathrm{T}}(k)+A(k) P(k) A^{\mathrm{T}}(k) \\
+\rho L(k) C(k) P(k) C^{\mathrm{T}}(k) L^{\mathrm{T}}(k) \\
-\rho L(k) C(k) P(k) A^{\mathrm{T}}(k) L^{\mathrm{T}}(k) \\
-\rho A(k) C(k) P(k) C^{\mathrm{T}}(k) L^{\mathrm{T}}(k), \\
P(0)=I
\end{gathered}
$$

turns to (30).

Next, we discuss the stability of the proposed FDF. In fact, by introducing $w(k)=\gamma(k)-\rho$ satisfying

$$
\begin{gathered}
\mathrm{E}\{w(k)\}=0, \\
\mathrm{E}\{w(i) w(j)\}=\varepsilon \delta_{i j},
\end{gathered}
$$

where $\varepsilon=\rho-\rho^{2}$ and $\delta_{i j}$ means the Kronecker delta function, an artificial stochastic backward control system associated with (24) can be defined as follows:

$$
\begin{gathered}
x_{a}(k-1)=\left(\bar{A}_{e}(k)+w(k) A_{e w}(k)\right)^{\mathrm{T}}(k) x_{a}(k) \\
+\left(\bar{C}_{e}(k)+w(k) C_{e w}(k)\right)^{\mathrm{T}}(k) u_{a}(k), \\
x_{a}(N)=0,
\end{gathered}
$$

where $\bar{A}_{e}(k)=A(k)-\rho L(k) C(k), A_{e w}(k)=L(k) C(k)$, $\bar{C}_{e}(k)=\rho V(k) C(k)$, and $C_{e w}(k)=V(k) C(k)$. From [36], if Assumptions 2 and 3 hold, there exists a unique positive solution $P(\cdot)$ to $(30)$ in the sense of finding a linear quadratic (LQ) control law $u_{a}^{*}(k)$ to (43). Furthermore, following [42], the proposed FDF is ESMS. This completes the proof.

Theorem 12 addresses a static solution to the two-objective optimization problem (9). A more generalized dynamic operator form of this theorem can be derived as follows.

Theorem 13. Let $L(k)$ be any observer gain that guarantees the stability of the proposed FDF (4); then $V_{o}(k)$ that maps $v(k) \mapsto$ $r(k)$ in the following way presents a dynamic post-filter for the auxiliary FDF design problem:

$$
\begin{aligned}
\xi_{o}(k+1)= & \left(A(k)-L_{o}(k) \gamma(k) C(k)\right) \xi_{o}(k) \\
& +\left(L(k)-L_{o}(k)\right) \nu(k), \\
r(k)= & V_{o}(k)\left(\gamma(k) C(k) \xi_{o}(k)+\nu(k)\right),
\end{aligned}
$$

where $L_{o}$ and $V_{o}$ are determined in (28) and (30).

Proof. The proof is straightforward as a result of Lemma 10. Hence, it is omitted here.

Theorem 12 can be readily extended to the stationary FDF design over an infinite horizon. In this case, the system matrices $A(k), B(k), B_{f}(k), B_{d}(k), C(k)$, and $D_{d}(k)$ will be replaced by constant matrices $A, B, B_{f}, B_{d}, C$, and $D_{d}$, respectively. The following result, which is the stationary version of Theorem 12 for LTI systems with measurement packet dropping, can be obtained by redefining $\mathbb{V}\left(\eta_{a}(k)\right)=$ $\eta_{a}^{\mathrm{T}}(k) P \eta_{a}(k)$ in Lemma 11 with a constant $P$ and replacing the Riccati difference equation (30) by a corresponding algebraic Riccati equation. In addition, from [24, 42], the ESMS of the proposed FDF can be guaranteed when Assumptions 2 and 3 are satisfied.

Corollary 14. Consider system (1) with constant system matrices and zero initial state. If Assumptions 2 and 3 hold, then the following matrix pair

$$
\begin{gathered}
L_{o}=\left(\rho A P C^{\mathrm{T}}+B_{d} D_{d}^{\mathrm{T}}\right) R^{-1}, \\
V_{o}=R^{-1 / 2}
\end{gathered}
$$

with

$$
R=\rho C P C^{\mathrm{T}}+D_{d} D_{d}^{\mathrm{T}}>0
$$


give a solution to (9) in infinite horizon and $P$ is the solution to the following equation:

$$
P=A P A^{\mathrm{T}}-L R L^{\mathrm{T}}+B_{d} B_{d}^{\mathrm{T}} .
$$

Remark 15. Theorem 12 gives unified analytical solutions to the associated FDF design problem of optimizing the defined stochastic $H_{-} / H_{\infty}$ and $H_{\infty} / H_{\infty}$ performance indices. It can easily conclude the following.

(i) If $\rho \neq 1$; that is, there measurement packet dropping occurs, the solution to the FDF design problem is derived based on a modified Riccati equation (30).

(ii) If $\rho=1$; that is, there is no measurement packet dropout, our results will coincide with the one given in $[18,19]$, where the modified Riccati equation (30) will reduce to a standard Kalman-like filtering Riccati equation.

Remark 16. Roughly speaking, the measurement packet dropping phenomenon depicted by the Bernoulli random variable can be divided into three categories, that is, measurement packet dropouts, stochastic finite step delay, and multiple packet dropouts [20]. It can be concluded that if the packet dropouts indicator (as $\gamma(k)$ in this paper) is online known, our proposed algorithm can be applied to these scenarios.

Remark 17. It should be pointed out that for system (1), the existing $H_{\infty}$ filtering-based fault estimation approach in [39] cannot achieve the fault detection goal since the fault distribution matrix in the measurement $y(k)$ is zero. However, our solution is independent on the fault distribution matrices, which infers its more generalized availability. In addition, comparing to the LMI-based $H_{\infty}$ fault estimation approaches given in [26-29], the proposed results are in analytical form and load less computational burden.

\section{Numerical Example}

To illustrate the effectiveness of the proposed method, we consider (1) with the following parameter matrices:

$$
\begin{gathered}
A(k)=\left[\begin{array}{ccc}
0.8 & 0.6 & 0 \\
0 & 0.5 & 0.5 \\
0 & 0 & 0.7
\end{array}\right], \quad B_{d}(k)=\left[\begin{array}{c}
1.3 \\
0.5 \\
1
\end{array}\right], \\
B_{f}(k)=\left[\begin{array}{c}
0.7 \\
1 \\
0.8
\end{array}\right], \quad C(k)=\left[\begin{array}{lll}
-0.5 & 1.5 & 0
\end{array}\right], \\
D_{d}(k)=0.5 .
\end{gathered}
$$

The missing measurement rate $\gamma(k)$ is displayed in Figure 1 with mean $\rho=0.8$ and the unknown input $d(k)$ is simulated in Figure 2. A stepwise fault signal is assumed to be as follows:

$$
f(k)= \begin{cases}1, & k \in[31,60] \\ 0, & \text { otherwise }\end{cases}
$$

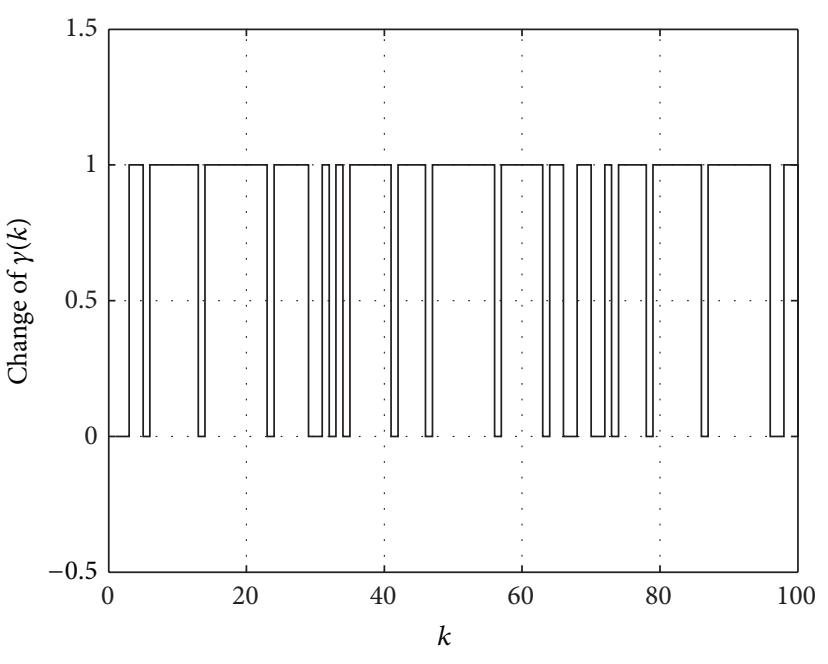

Figure 1: Change of $\gamma(k)$.

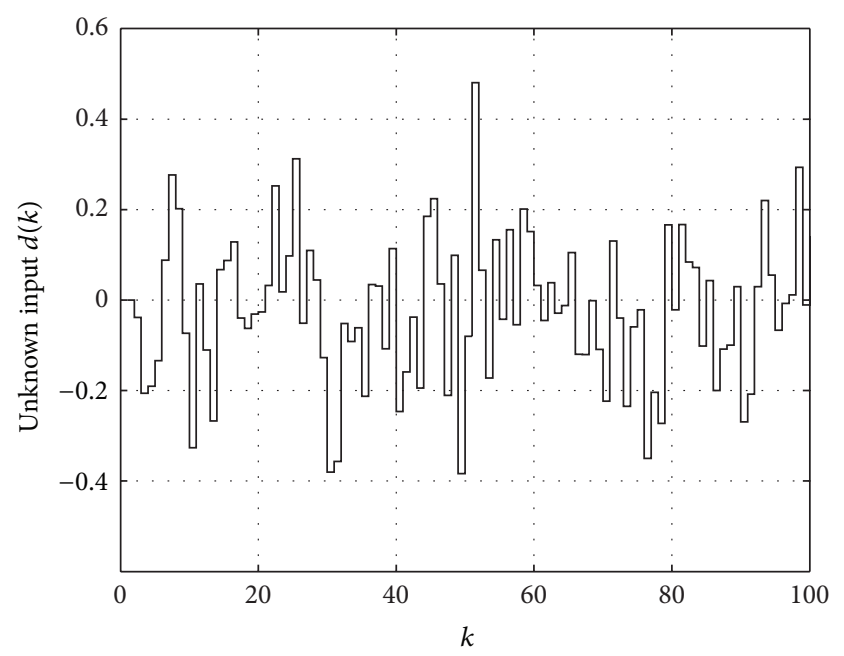

FIgURE 2: Unknown input $d(k)$.

Set $P(0)=I$. We design the FDF by directly applying Theorem 12. The generated residual $r(k)$ is shown in Figure 3 and its residual evaluation function $J(k)$ in (10) is displayed in Figure 4 with the corresponding threshold $J_{\text {th }}=0.7457$. It can be seen from the simulation results that $J(32)=2.2504>J_{\text {th }}$ which implies that the residual can deliver fault alarm in 1 second after it occurs.

\section{Conclusions}

In this paper, the problem of FD for LDTV systems with measurement packet dropping has been dealt with. Under the condition that the packet dropout indicator is on-line available, an observer-based FDF has been employed as a residual generator through exploiting the information brought by the packet dropout indicator. The design of FDF has been formulated in the framework of maximizing stochastic $H_{-} / H_{\infty}$ or $H_{\infty} / H_{\infty}$ performance index. An analytical optimal solution 


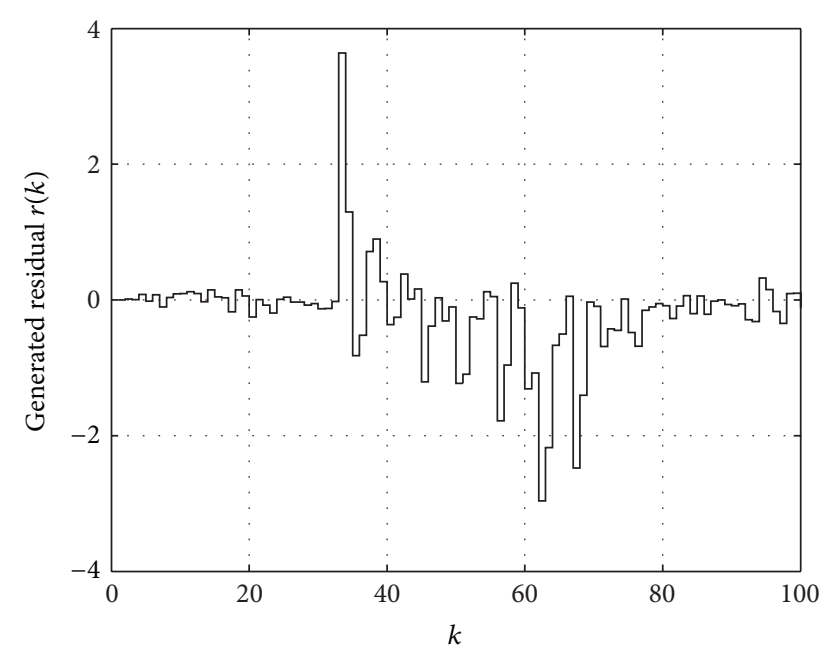

FIgURE 3: Residual $r(k)$.

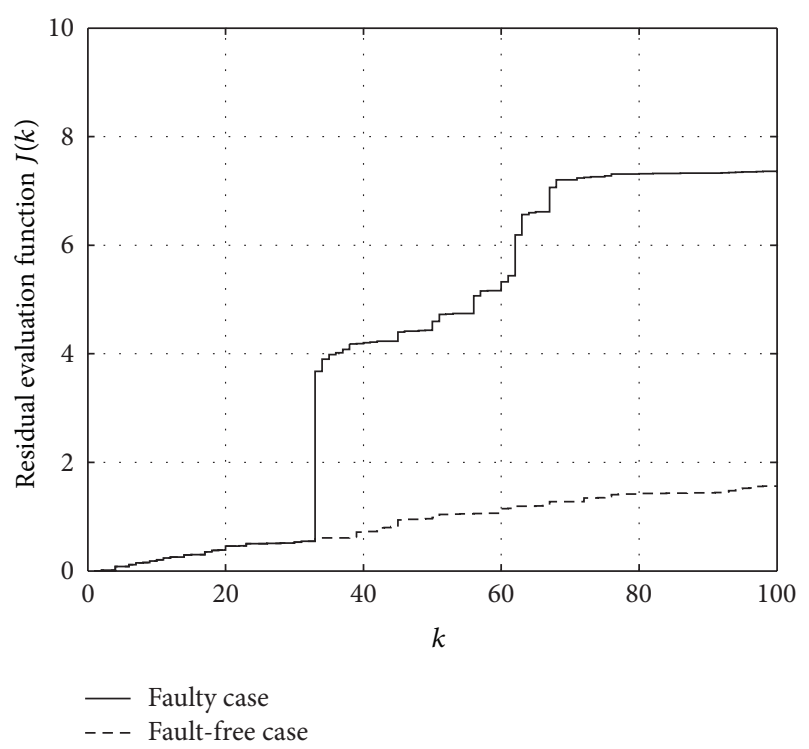

Figure 4: Residual evaluation function $J(k)$.

has been derived by solving a modified Riccati equation based on the proposed adjoint operator optimization method. The achieved result has been illustrated by a numerical example.

\section{Acknowledgments}

This work is partially supported by National Natural Science Foundation of China (no. 61203083 and no. 61074021), the Doctoral Foundation of University of Jinan (no. XBS1242).

\section{References}

[1] J. Chen and R. Patton, Robust Model-Based Fault Diagnosis for Dynamic Systems, Kluwer Academic Publishers, Boston, Mass, USA, 1999.

[2] S. X. Ding, Model-Based Fault Diagnosis Techniques, Springer, Berlin, Germany, 2008.
[3] B. Kulcsár and M. Verhaegen, "Robust inversion based fault estimation for discrete-time LPV systems," IEEE Transactions on Automatic Control, vol. 57, no. 6, pp. 1581-1586, 2012.

[4] Z. Wang, H. Guan, and C. Zheng, "Fault diagnosis observer design for discrete-time delayed complex interconnected networks with linear coupling," Mathematical Problems in Engineering, vol. 2012, Article ID 860489, 22 pages, 2012.

[5] J. Yu, G. Sun, and H. R. Karimi, "Fault-reconstruction-based cascaded sliding mode observers for descriptor linear systems," Mathematical Problems in Engineering, vol. 2012, Article ID 623426, 20 pages, 2012.

[6] Y. Yin, P. Shi, F. Liu, and J.-S. Pan, "Gain-scheduled fault detection on stochastic nonlinear systems with partially known transition jump rates," Nonlinear Analysis. Real World Applications, vol. 13, no. 1, pp. 359-369, 2012.

[7] L. Wu, X. Yao, and W. X. Zheng, "Generalized $H_{2}$ fault detection for two-dimensional Markovian jump systems," Automatica, vol. 48, no. 8, pp. 1741-1750, 2012.

[8] J. Chen and R. Patton, "Standard $H_{\infty}$ filtering formulation of robust fault detection," in Proceedings of SAFEPROCESS, Budapest, Hungary, 2000.

[9] M.-Y. Zhong, S. Liu, and H.-H. Zhao, "Krein space-based $H_{\infty}$ fault estimation for linear discrete time-varying systems," Acta Automatica Sinica, vol. 34, no. 12, pp. 1529-1533, 2008.

[10] Y. Li and M. Zhong, "Fault detection filter design for linear discrete time-varying systems with multiplicative noise," Journal of Systems Engineering and Electronics, vol. 22, no. 6, pp. 982-990, 2011.

[11] H. Dong, Z. Wang, and H. Gao, "Fault detection for Markovian jump systems with sensor saturations and randomly varying nonlinearities," IEEE Transactions on Circuits and Systems I, vol. 59, no. 10, pp. 2354-2362, 2012.

[12] S. X. Ding, T. Jeinsch, P. M. Frank, and E. L. Ding, "A unified approach to the optimization of fault detection systems," International Journal of Adaptive Control and Signal Processing, vol. 14, no. 7, pp. 725-745, 2000.

[13] J. Wang, G. Yang, and J. Liu, "An LMI approach to $H_{-}$index and mixed $H_{-} / H_{\infty}$ fault detection observer design," Automatica, vol. 43, no. 9, pp. 1656-1665, 2008.

[14] A. Casavola, D. Famularo, and G. Franzè, "Robust fault detection of uncertain linear systems via quasi-LMIs," Automatica, vol. 44, no. 1, pp. 289-295, 2008.

[15] P. Zhang, S. X. Ding, G. Z. Wang, and D. H. Zhou, "Fault detection of linear discrete-time periodic systems," IEEE Transactions on Automatic Control, vol. 50, no. 2, pp. 239-244, 2005.

[16] P. Zhang, S. X. Ding, and P. Liu, "A lifting based approach to observer based fault detection of linear periodic systems," IEEE Transactions on Automatic Control, vol. 57, no. 2, pp. 457-462, 2012.

[17] X. Li, Fault Detection Filter Design for Linear Systems [Ph.D. thesis], Louisiana State University, 2009.

[18] M. Zhong, S. X. Ding, and E. L. Ding, "Optimal fault detection for linear discrete time-varying systems," Automatica, vol. 46, no. 8, pp. 1395-1400, 2010.

[19] M. Zhong and S. X. Ding, "A projection-based method of fault detection for linear discrete time-varying system," International Journal of Systems Science, 2011.

[20] J. Hespanha, P. Naghshtabrizi, and Y. Xu, "A survey of recent results in networked control systems," Proceedings of the IEEE, vol. 95, no. 1, pp. 138-162, 2007. 
[21] L. Bakule and M. de la Sen, "Decentralized resilient $H_{\infty}$ observer-based control for a class of uncertain interconnected networked systems," in Proceedings of the American Control Conference, Baltimore, Md, USA, 2010.

[22] K. You, M. Fu, and L. Xie, "Mean square stability for Kalman filtering with Markovian packet losses," Automatica, vol. 47, no. 12, pp. 2647-2657, 2011.

[23] Y. Mo and B. Sinopoli, "Kalman filtering with intermittent observations: tail distribution and critical value," IEEE Transactions on Automatic Control, vol. 57, no. 3, pp. 677-689, 2012.

[24] H. Zhang, X. Song, and L. Shi, "Convergence and mean square stability of suboptimal estimator for systems with measurement packet dropping," IEEE Transactions on Automatic Control, vol. 57, no. 5, pp. 1248-1253, 2012.

[25] Y. Zhang, C. Liu, and X. Mu, "On stochastic finite-time control of discrete-time fuzzy systems with packet dropout," Discrete Dynamics in Nature and Society, vol. 2012, Article ID 752950, 18 pages, 2012.

[26] H. Gao, T. Chen, and L. Wang, "Robust fault detection with missing measurements," International Journal of Control, vol. 81, no. 5, pp. 804-819, 2008.

[27] Y. Zhao, J. Lam, and H. Gao, "Fault detection for fuzzy systems with intermittent measurements," IEEE Transaction on Fuzzy Systems, vol. 17, no. 2, pp. 398-410, 2009.

[28] X. Yao, L. Wu, and W. X. Zheng, "Fault detection filter design for Markovian jump singular systems with intermittent measurements," IEEE Transactions on Signal Processing, vol. 59, no. 7, pp. 3099-3109, 2011.

[29] D. Du, B. Jiang, and P. Shi, "Fault detection for discrete-time switched systems with intermittent measurements," International Journal of Control, vol. 85, no. 1, pp. 78-87, 2012.

[30] X. He, Z. Wang, Y. Ji, and D. Zhou, "Robust fault detection for networked systems with distributed sensors," IEEE Transaction on Aerospace and Electronic Systems, vol. 47, no. 1, pp. 166-177, 2008.

[31] X. He, Z. Wang, and D. H. Zhou, "Robust fault detection for networked systems with communication delay and data missing," Automatica, vol. 45, no. 11, pp. 2634-2639, 2009.

[32] D. Zhou, X. He, Z. Wang et al., "Leakage fault diagnosis for an internet-based three-tank system: an experimental study," IEEE Transaction on Control Systems Technology, vol. 20, no. 4, pp. 857-870, 2011.

[33] X. Wan and H. Fang, "Fault detection for networked nonlinear systems with time delays and packet dropouts," Circuits, Systems, and Signal Processing, vol. 31, no. 1, pp. 329-345, 2012.

[34] X. Wan, H. Fang, and F. Yang, "Fault detection for a class of networked nonlinear systems subject to imperfect measurements," International Journal of Control, Automation, and Systems, vol. 10, no. 2, pp. 265-274, 2012.

[35] W. Zhang, Y. Huang, and L. Xie, "Infinite horizon stochastic $\mathrm{H}_{2} / \mathrm{H}_{\infty}$ control for discrete-time systems with state and disturbance dependent noise," Automatica, vol. 44, no. 9, pp. 23062316, 2008.

[36] Y. Huang, W. Zhang, and H. Zhang, "Infinite horizon LQ optimal control for discrete-time stochastic systems," in Proceedings of the 6th World Congress on Intelligent Control and Automation, Dalian, China, 2006.

[37] M. Green and D. J. N. Limebeer, Linear Robust Control, Prentice Hall, Upper Saddle River, NJ, USA, 1995.

[38] X. Li and K. Zhou, "A time domain approach to robust fault detection of linear time-varying systems," Automatica, vol. 45, no. 1, pp. 94-102, 2009.
[39] Y. Li and M. Zhong, "On designing robust $H_{\infty}$ fault detection filter for linear discrete time-varying systems with multiple packet dropouts," Acta Automatica Sinica, vol. 36, no. 12, pp. 1788-1796, 2010

[40] E. Gershon, U. Shaked, and I. Yaesh, " $H_{\infty}$ control and filtering of discrete-time stochastic systems with multiplicative noise," Automatica, vol. 37, no. 3, pp. 409-417, 2001.

[41] A. Bobrowski, Functional Analysis for Probability and Stochastic Processes. An Introduction, Cambridge University Press, Cambridge, UK, 2005.

[42] V. Dragan, T. Morozan, and A.-M. Stoica, Mathematical Methods in Robust Control of Discrete-Time Linear Stochastic Systems, Springer, New York, NY, USA, 2010. 


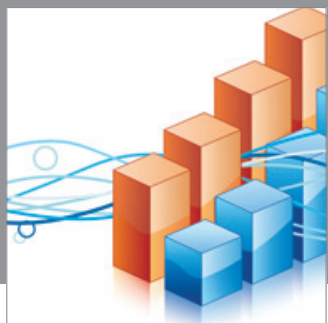

Advances in

Operations Research

mansans

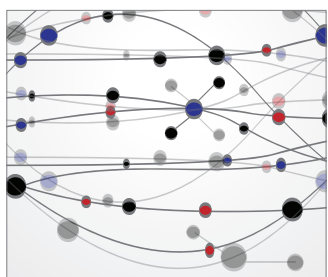

The Scientific World Journal
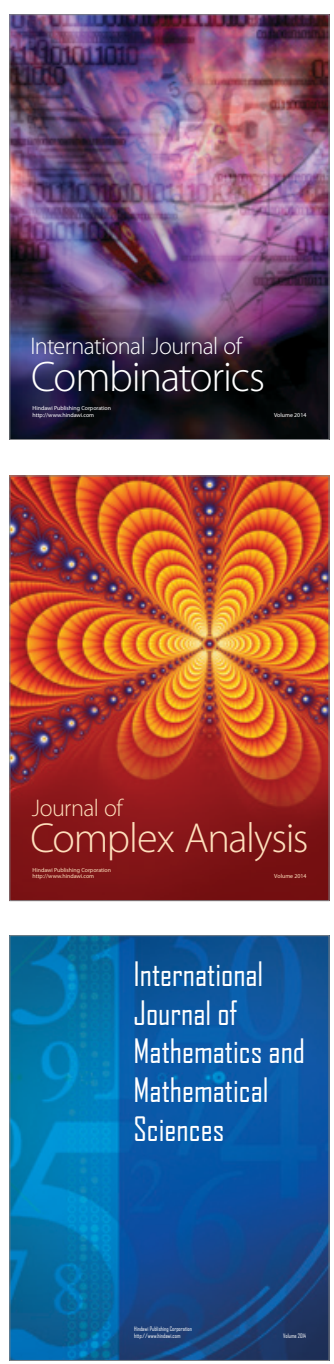
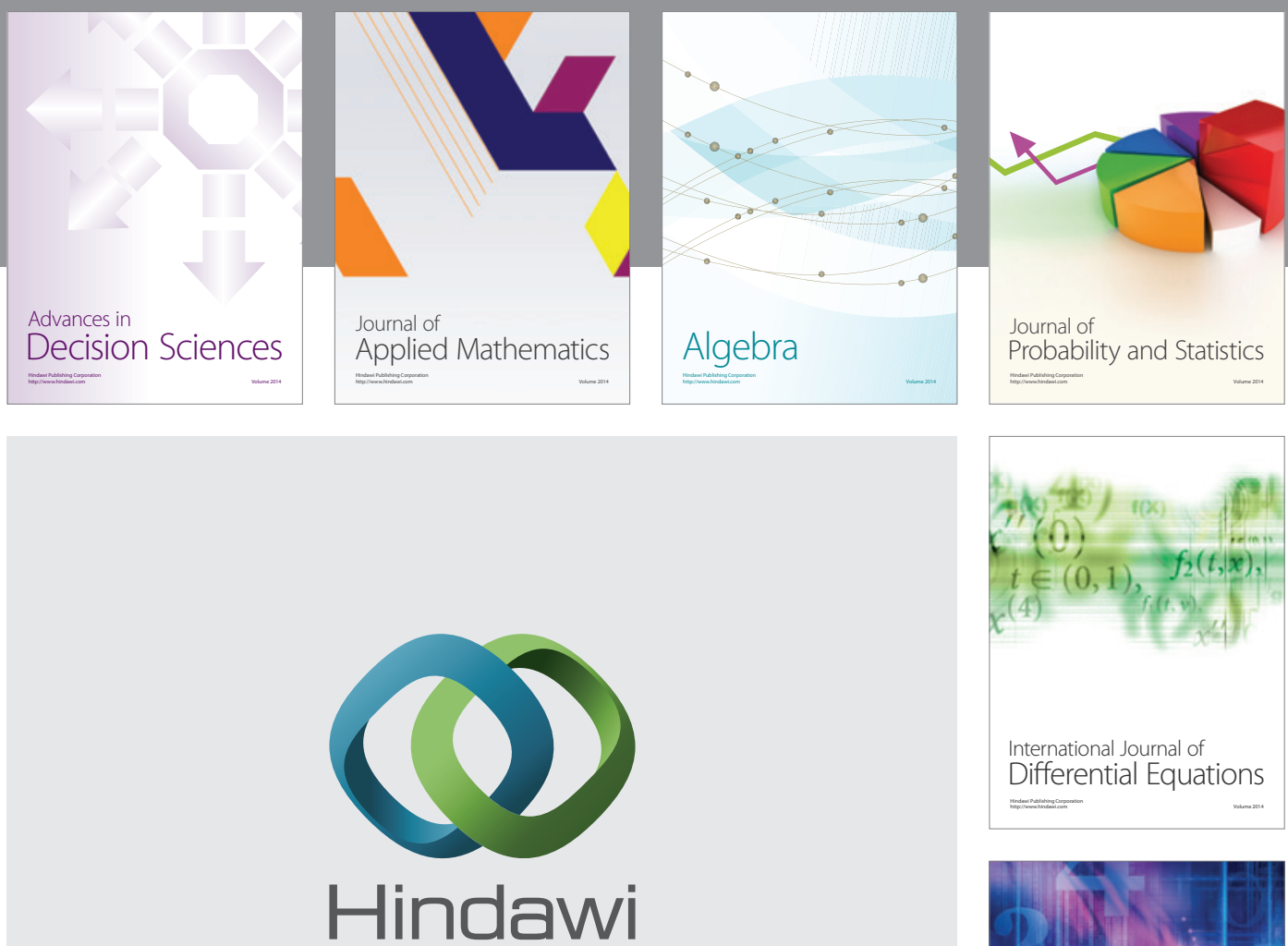

Submit your manuscripts at http://www.hindawi.com
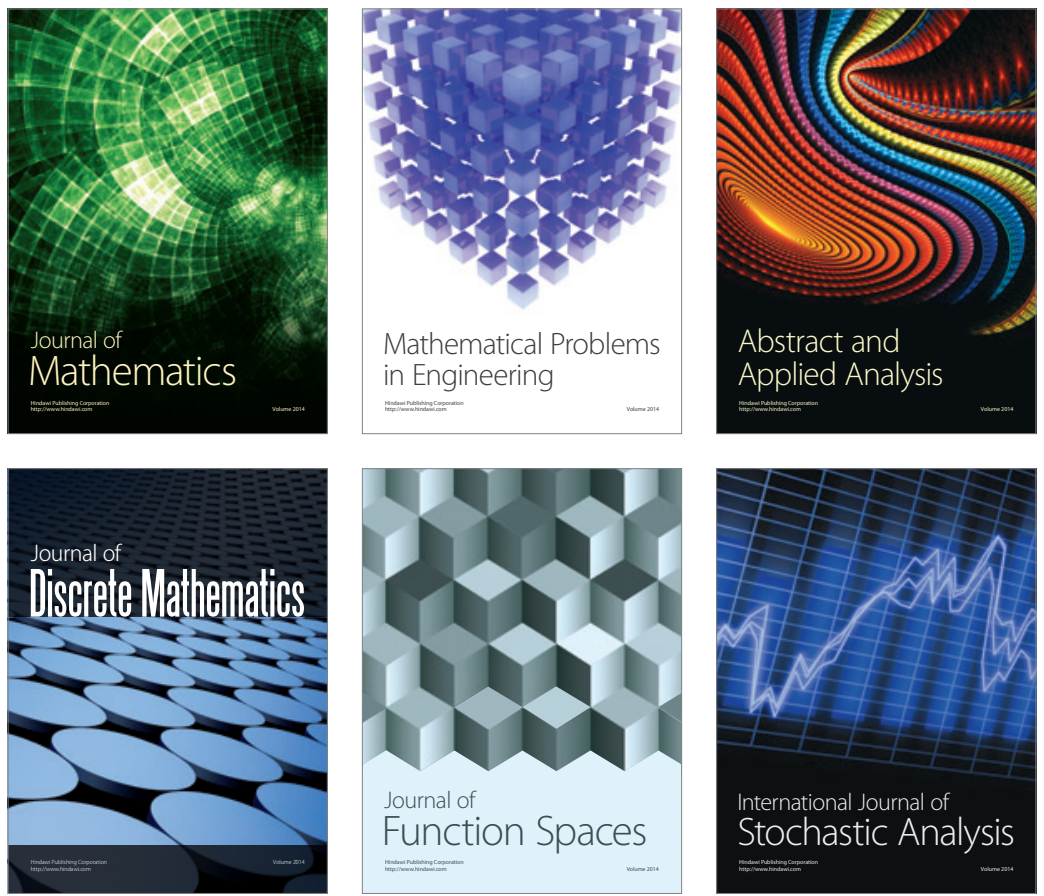

Journal of

Function Spaces

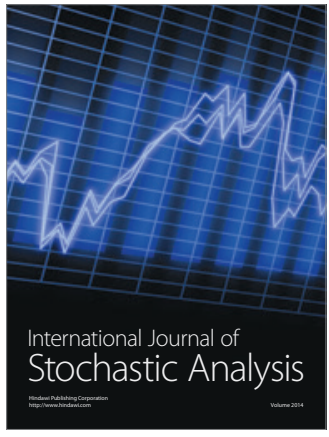

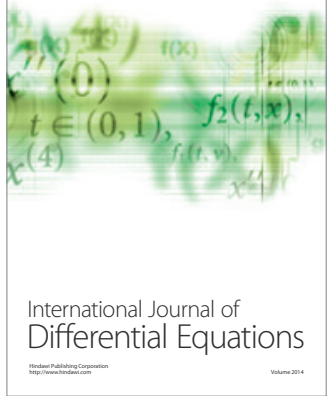
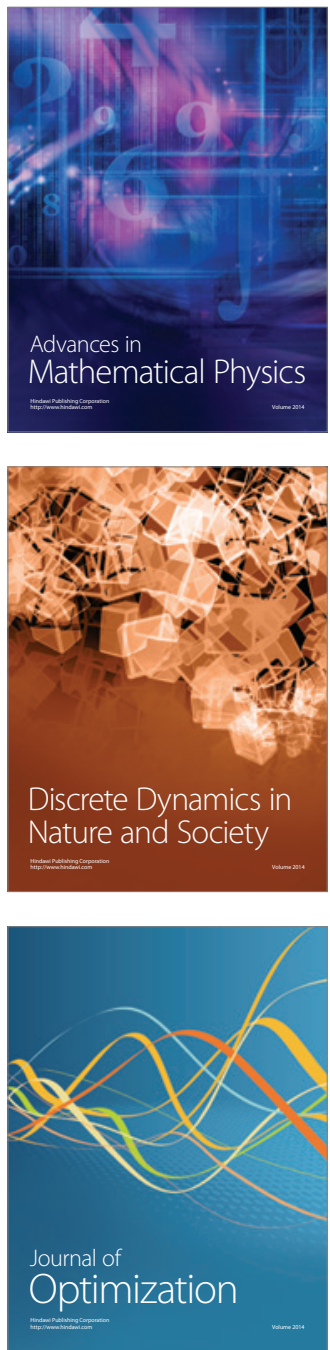